\title{
A experiência da reorganização da Atenção Primária à Saúde - APS e trabalho dos agentes comunitários de saúde frente à COVID-19 em um município no interior do Amazonas
}

The experience of the reorganization of primary health care and the work of community health workers in front of COVID-19 in a municipality in the interior of Amazonas

La experiencia de la reorganización de la atención primaria de salud y el trabajo de los agentes de salud comunitarios frente a COVID-19 en un municipio en el interior de Amazonas

Mayline Menezes da MATA ${ }^{(1)}$ Daiane Nascimento de CASTRO ${ }^{(1)}$ Cléber Araújo GOMES ${ }^{(1)}$ Juliberta Alves de MACÊDO(1) Maria Helena Ribeiro De $\mathrm{CHECCHI}^{(1)}$ Abel Santiago Muri GAMA ${ }^{(1)}$ Luís Paulo Souza e SOUZA ${ }^{(1)}$

Recebido: 12 jul 2020 Revisado: 8 set 2020 Aceito: 16 set 2020

Autor de correspondência: Mayline Menezes da Mata mayline.damata@gmail.com

Conflito de interesses: Os autores declaram não haver nenhum interesse profissional ou pessoal que possa gerar conflito de interesses em relação a este manuscrito.

\footnotetext{
(1)Universidade Federal do Amazonas - UFAM, Instituto de Saúde e Biotecnologia, Coari, AM Brasil.
}

\section{Resumo}

A COVID-19 atingiu o Amazonas, ampliando as vulnerabilidades que já existiam, principalmente nas cidades do interior. Considerando o importante papel que a Atenção Primária à Saúde - APS tem diante da atual pandemia, além do fato de que maior parte dos serviços de saúde nos municípios do interior do estado é ofertada pela APS, buscouse descrever a experiência da reorganização da APS, com ênfase nas ações dos agentes comunitários $(\mathrm{AC})$ de um município do interior do Amazonas. Coari está localizado na mesorregião Centro Amazonense, cujo acesso é por via fluvial e aérea, a 363 quilômetros da capital, Manaus. Até o dia 12 de setembro de 2020, o município ocupava a segunda posição no estado com maior número de casos ( $n=6.990)$; e terceiro em número de óbitos $(n=105)$. Dispõe de 223 ACS, integrando 22 equipes da Estratégia Saúde da Família e três Núcleos Ampliados de Saúde da Família e Atenção Básica. Na pandemia, 35 ACS foram afastados por estarem nos grupos de risco. Destaca-se que os ACS da zona urbana e rural (incluindo as comunidades ribeirinhas) incorporaram ao seu rol de atividades ações de monitoramento dos casos confirmados e suspeitos, por meio de visitas domiciliares ou remotamente (por celular). Destacam-se os desafios advindos das barreiras geográficas e de sinal telefônico, características das moradias (alta densidade domiciliar), elevadas prevalências de outras doenças comuns na região, e deficiência de profissionais de nível superior nas unidades da zona rural/ribeirinha. Contudo, mesmo com os desafios, os ACS têm sido atores estratégicos para o enfrentamento da pandemia em Coari, atuando na organização do serviço e na continuidade do cuidado. Estratégias que fortaleçam a APS, colocando-a no centro das discussões, priorizando recursos para seu financiamento e reconhecendo o protagonismo dos ACS, são essenciais para o sucesso do enfrentamento 
à COVID-19 no país, principalmente nos municípios do interior.

Descritores: Infecções por Coronavírus; Agentes Comunitários de Saúde; Estratégia Saúde da Família; Atenção Primária à Saúde; População Rural.

\begin{abstract}
The COVID-19 hit the Amazon, expanding the vulnerabilities that already existed, especially in the cities of the interior. Considering the important role that Primary Health Care - PHC has in the current pandemic, besides the fact that most health services in the municipalities of the interior of the state are offered by $\mathrm{PHC}$, we sought to describe the experience of the reorganization of $\mathrm{PHC}$, with emphasis on the actions of Community Health Workers (CHW) of a municipality in the interior of Amazonas. Coari is located in the mesoregion Centro Amazonense, whose access is by river and air, 363 kilometers from the capital, Manaus. Until September 12, 2020, the municipality occupied the second position in the state with the highest number of cases $(n=6,990)$; and third in number of deaths $(n=105)$. It has $223 \mathrm{CHW}$, integrating 22 family health strategy teams and three expanded Centers of Family Health and Primary Care. In the pandemic, $35 \mathrm{CHW}$ were removed because they were in the risk groups. It's noteworthy that the $\mathrm{CHW}$ of the urban and rural area (including riverside communities) incorporated into their list of activities actions to monitor confirmed and suspected cases, through home visits or remotely (by mobile phone). We highlight the challenges arising from geographical barriers and telephone signal, characteristics of housing (high home density), high prevalence of other common diseases in the region, and deficiency of higher education professionals in rural/riverside units. However, even with the challenges, the $\mathrm{CHW}$ have been strategic actors to cope with the pandemic in Coari, acting in the organization of the service and in the continuity of care. Strategies that strengthen $\mathrm{PHC}$, placing it at the center of discussions, prioritizing resources for its financing and recognizing the role of $\mathrm{CHW}$, are essential for the success of coping with COVID-19 in the country, especially in the municipalities of the interior.
\end{abstract}

Keywords: Coronavirus Infections; Community Health Workers; Family Health Strategy; Primary Health Care; Rural Population.

\title{
Resumen
}

COVID-19 alcanzó el Amazonas, ampliando las vulnerabilidades que ya existían, especialmente en las ciudades del interior. Teniendo en cuenta el importante papel que la Atención Primaria de Salud PHC tiene en la pandemia actual, además del hecho de que la mayoría de los servicios de salud en los municipios del interior del estado son ofrecidos por la PHC, buscamos describir la experiencia de la reorganización de la PHC, con énfasis en las acciones de los Agentes Comunitarios de Salud (ACS) de un municipio en el interior de Amazonas. Coari se encuentra en la mesorregion Centro Amazonense, cuyo acceso es por río y aire, a 363 kilómetros de la capital, Manaus. Hasta el 12 de septiembre de 2020, el municipio ocupó la segunda posición en el estado con el mayor número de casos $(n=6.990)$; y tercero en número de muertes $(n=105)$. Cuenta con 223 ACS, integrando 22 equipos de estrategia de salud familiar y tres Centros ampliados de Salud Familiar y Atención Primaria. En la pandemia, se eliminaron 35 ACS porque estaban en los grupos de riesgo. Cabe destacar que los ACS de la zona urbana y rural (incluidas las comunidades ribereñas) incorporó a su lista de actividades acciones para vigilar los casos confirmados y sospechosos, a través de visitas domiciliarias o de forma remota (por teléfono móvil). Destacamos los desafíos derivados de las barreras geográficas y la señal telefónica, las características de la vivienda (alta densidad de viviendas), la alta prevalencia de otras enfermedades comunes en la región y la deficiencia de los profesionales de la educación superior en las unidades rurales/ribereñas. Sin embargo, incluso con los desafíos, el ACS ha sido actores estratégicos para hacer frente a la pandemia en Coari, actuando en la organización del servicio y en la continuidad de la atención. Las estrategias que fortalecen la PHC, situándola en el centro de los debates, priorizando los recursos para su financiamiento y reconociendo el papel de ACS, son esenciales para el éxito de hacer frente a COVID-19 en el país, especialmente en los municipios del interior.

Palabras-claves: Infecciones por Coronavirus; Agentes Comunitarios de Salud; Estrategia de Salud Familiar; Atención Primaria de Salud; Población Rural. 


\section{Introdução}

ISSN 2179-6750

Em fevereiro de 2020, o Brasil passou a compor a lista de países afetados pela pandemia da COVID-19 e, atualmente, ocupa a terceira posição mundial em número de casos e óbitos em 14 de setembro, correspondendo a 4.345 .610 e 132.006 , respectivamente. ${ }^{1}$ Diante das dimensões continentais e das expressivas desigualdades sociais e sanitárias, a doença se distribui de forma diferente entre os estados brasileiros. Dentre eles, ressalta-se o Amazonas, marcado historicamente por iniquidades nas condições de vida, educação e saúde das populações, figurando-se com os piores indicadores de morbimortalidade pelo novo por coronavírus no país. ${ }^{1,2}$ Ao considerar o contexto do interior, o cenário de disparidades mostra-se ainda mais complexo, especialmente no setor saúde, o qual possui inúmeras barreiras de acesso, bem como indisponibilidade de serviços e profissionais. $^{3}$

As estratégias para o enfrentamento da COVID-19 exigem articulação intersetorial e, no tocante aos serviços de saúde, demanda intensa reorganização em todos os níveis de atenção. Neste sentido, a Atenção Primária à Saúde - APS e a Estratégia Saúde da Família ESF, consideradas espinhas dorsais na reordenação Sistema Único de Saúde - SUS, têm sido destacadas como fundamentais para controle da pandemia. ${ }^{4}$ A APS passou por importantes mudanças como a reorganização do acesso, fluxos de atendimento, inovação nas ferramentas de monitoramento e acompanhamento dos pacientes, bem como a redefinição de papéis dos profissionais e ações das equipes. ${ }^{5}$

Neste contexto, os Agentes comunitários de saúde (ACS) exercem um papel fundamental no enfrentamento da COVID-19 junto às equipes das ESF's e dos Núcleos Ampliados de Saúde da Família e Atenção Básica - NASF-AB. O trabalho destes profissionais consiste no apoio à identificação e busca ativa de casos confirmados e/ou suspeitos, além de orientar a comunidade quanto às medidas de prevenção e quanto às condutas diante do agravamento da situação de saúde. ${ }^{6}$

Apesar das recomendações apresentadas pelo Ministério da Saúde, sabe-se que a organização da APS se deu de variadas formas em todo o território nacional, fazendo-se necessária a caracterização e discussão das experiências locais e suas implicações na atenção à saúde. Assim, o presente artigo objetiva descrever a experiência da reorganização da APS, com ênfase nas ações dos Agentes Comunitários de um município do interior do Amazonas.

\section{Metodologia}

Trata-se de um relato de experiência da reorganização da APS, com ênfase nas ações dos ACS no município de Coari, baseado em documentos oficiais produzidos pelo município. 
Localizado na mesorregião Centro Amazonense, a 363 quilômetros da capital, Manaus, sem acesso terrestre. A população estimada para o ano de 2019 foi de 85.097 habitantes, ${ }^{7}$ sendo que um terço vive em área rural. Apresenta um baixo Índice de Desenvolvimento Humano Municipal - IDHM $(0,586)^{7}$ e alta densidade domiciliar (as residências apresentam dois a três cômodos e abrigam, em média, seis moradores). O município possui 208 comunidades ribeirinhas, com acesso principal por via fluvial e deslocamento que podem durar minutos ou dias de navegação. ${ }^{8}$ Vivem de atividades extrativistas (animal e vegetal), agricultura familiar e subsídios provenientes de programas sociais governamentais; além disso, a maioria das comunidades não dispõem de acesso à serviços de saneamento básico, de assistência à saúde e de comércio, uma vez que estes concentram-se na área urbana.

Até o dia 12 de setembro de 2020, o município ocupava a segunda posição no ranque do estado com maior número de casos da doença $(n=6.990)$, estando atrás apenas da capital do estado, Manaus $(n=45.327)$. No tocante ao número de óbitos, ocupava a terceira colocação no ranque dos municípios do interior, com 105 registros, superado pelos municípios Manacapuru $(n=153)$ e Parintins $(n=120){ }^{9}$

\section{Resultados e Discussão}

A APS do município conta com 14 unidades de saúde da família (USF), das quais uma é referência para as comunidades ribeirinhas (localizada na área urbana) e uma é unidade fluvial. Dispõe de 22 equipes de ESF e 223 ACS, distribuídos entre área urbana $(n=105)$ e rural $(n=118)$. A cobertura da ESF alcança 97,3\% do município e, no apoio matricial, Coari possui três equipes de NASF (eNASF) na modalidade 1, composto por fisioterapeuta, assistente social, fonoaudiólogo, nutricionista, educador físico e psicólogo. ${ }^{10}$ Cada eNASF está vinculada a cinco equipes de Saúde da Família, cobrindo 68\% das eSF do município.

\section{Reorganização dos fluxos nas unidades}

Para o enfrentamento da pandemia, assim como toda a rede de saúde, a APS do município foi reconfigurada. Uma das primeiras medidas adotadas foi definir duas unidades de saúde da área urbana como referência para o atendimento de síndromes respiratórias/gripais ou usuários com sintomas respiratórias, com atendimento ampliado 12 horas por dia (de $7 \mathrm{~h}$ às 19h), de segunda a segunda; contratação de novos profissionais. Em ambas as unidades, todos os demais atendimentos de rotina foram suspensos, sendo realizado, portanto, apenas atendimento para casos suspeitos e confirmados de COVID-19.

$\mathrm{Na}$ frente de cada unidade foi montada uma barreira física com um profissional paramentado com equipamentos de proteção individual (EPI) para recebimento dos 
usuários, direncionando-os à recepção para elaboração de ficha, triagem (aferição do peso, estatura, pressão arterial e temperatura) e atendimento médico. Em caso de suspeita de COVID-19, era solicitado o teste rápido e prescrito medicação preventiva, disponíveis na unidade de referência. Caso os medicamentos estivessem em falta, o usuário era encaminhado à Central de Medicamentos do município. Posteriormente, o usuário era encaminhado para o atendimento de Enfermagem, procedendo com a notificação. Todas as unidades foram equipadas com os respectivos EPI, máscara N95, óculos de proteção, viseira, luva estéreo ou procedimento, máscara cirúrgica, avental, gorro e pro-pé e kits de higiene (sabão e/ou álcool em gel a 70\%). Destaca-se a realização contínua da desinfecção das unidades e sanitização total, uma vez por semana, por empresa especializada.

Nas demais unidades, permaneceram os serviços essenciais e presenciais, tais como consultas de pré-natal, puericultura, vacinação, acompanhamento de diabéticos e hipertensos, realização de testes rápidos, monitoramento via telefone dos casos positivos e suspeitos de COVID-19 (a partir de 5 de maio de 2020), além do atendimento à demanda espontânea. No escopo do NASF, as atividades individuais e coletivas foram suspensas nos primeiros meses de pandemia e houve redirecionamento dos profissionais para realização de monitoramento de casos suspeitos ou casos com sintomas leves a moderados conjuntamente à vigilância epidemiológica e unidades de referência.

No início da pandemia, o NASF atuou na prevenção através de ações informativas e educativas sobre higienização das mãos, uso de máscara, etiqueta respiratória, distanciamento social, dentre outros. Com a chegada de embarcações clandestinas no município, em abril, começaram a aparecer os primeiros casos de COVID-19. Assim, o NASF atuou no monitoramento das pessoas em quarentena e dos contaminados via remota, informando sobre a realização e agendamento dos testes de diagnóstico. Ao final de maio, foi contratada uma equipe própria para o combate à COVID-19, sendo que o NASF continuou apenas como suporte. Nos meses de agosto e setembro, com a implementação de uma lei que torna facultativa o modelo do NASF nos municípios, foi instituído pelo Ministério da Saúde o Programa Melhor em Casa, ${ }^{11}$ em que os antigos profissionais foram remanejados para novas funções.

Embora consista em um desafio diante da pandemia, a garantia e a importância da continuidade do cuidado na APS com a manutenção das atividades preventivas e curativas, têm sido amplamente discutidas. ${ }^{5}$ Enfatiza-se, portanto, como ponto positivo na reorganização da APS municipal, a continuidade das atividades essenciais nas unidades básicas, permitindo o acesso aos serviços para outras demandas de saúde. Por outro lado, observou-se a supressão dos serviços do NASF, que realizam ações preventivas e em grande parte clínico-assistenciais, sem a substituição por outras estratégias para assistência dos 
usuários. A não oferta das atividades de rotina ou descontinuidade do cuidado pode ocasionar aumento da morbimortalidade por outras doenças, agravando ainda mais os impactos da crise sanitária. ${ }^{12}$

Vale destacar o uso de ferramentas tecnológicas para a garantia das ações na APS, tais como teleatendimento e teleconsulta, que são relatados como principal alternativa adotada por diversos países e em experiências nacionais. ${ }^{13,14}$ No entanto, no município em questão, não foi utilizado tal recurso, colocando em cheque a realidade das áreas remotas e suas incontáveis dificuldades no uso destes recursos, tais como a indisponibilidade e/ou baixa qualidade de aparelhos, linhas telefônicas ou acesso à internet nas unidades e dos usuários, a questão da privacidade nos domicílios ou mesmo a capacidade de manuseio pelos pacientes.

É importante discutir, também, sobre a estratégia de estabelecer unidades exclusivas de atendimento à COVID-19 na APS e algumas desvantagens. Embora consista em uma medida para evitar a propagação da doença e prevenir a contaminação de outros usuários e profissionais, pode dificultar o deslocamento e acesso daqueles que buscam atendimento, comprometendo o diagnóstico e tratamento, além de desassistir os territórios para as demais ações de rotina. Em contraponto, ressalta-se os históricos desafios enfrentados pelas unidades de saúde que vão desde a inadequação de infraestrutura até o desabastecimento de recursos humanos e materiais, o que pode comprometer a preparação e adequação para atendimento aos casos suspeitos da doença. ${ }^{15}$

\section{Ações dos agentes comunitários de saúde (ACS)}

O trabalho dos ACS foi reorganizado conforme recomendações técnicas do Ministério da Saúde. ${ }^{16}$ Dos 223 ACS, 35 foram afastados inicialmente pela Secretaria Municipal de Saúde - SEMSA, por pertencerem ao grupo de risco à COVID-19; foram realizadas capacitações aos que continuaram na linha de frente e distribuição de EPI. A redução quantitativa dos ACS, sem as devidas substituições, é vista como preocupante, dada a situação crítica do município, com o aumento diário de casos confirmados, podendo impactar negativamente no enfrentamento da pandemia. ${ }^{9}$

Os ACS que integravam as equipes das unidades destinadas a serem de referência, foram direcionados às demais unidades, passando a atuar no planejamento da equipe, organização das agendas, atendimento telefônico e respondendo mensagens de aplicativos de comunicação dos usuários - como whatsapp.

Em relação às ações executadas pelos ACS no território, permaneceram visitas domiciliares (VD) a usuários com dificuldades motoras, acamados e aqueles que estão cadastrados no Sistema de Cadastramento e Acompanhamento de Hipertensos e Diabéticos 
(HIPERDIA), atuando, exclusivamente, na entrega de receituários médicos atualizados. Foi incluída a busca ativa dos usuários em situação de risco e vulnerabilidade quando não era possível contactá-los de forma remota. Para isso, os ACS adotaram medidas preventivas, como o uso de EPI e distanciamento físico, adentrando nos domicílios somente em casos de extrema necessidade. O distanciamento físico adotado pelos ACS é imprescindível, uma vez que o município apresenta alta densidade domiciliar, ou seja, um mesmo cômodo abriga várias pessoas, assim as VD (dentro do domicílio), aumentariam as chances de contágio pelo SARS-CoV-2.

Ressalta-se que todas as unidades foram equipadas com, no mínimo, um telefone móvel, com acesso à internet, vislumbrando a continuidade do cuidado, minimizando potenciais riscos tanto para os ACS, quanto aos usuários.

No atual cenário, a incorporação de ferramentas tecnológicas e o uso das redes sociais contribuíram positivamente para a continuidade do cuidado e fortalecimento do vínculo entre o serviço e a comunidade, sendo os ACS protagonistas desta ação. Cabe destacar que a implementação de tais ferramentas também apresentaram dificuldades relativas à falta de sinal telefônico em algumas áreas e de internet, sendo que uma parte considerável dos usuários não dispõe de telefone móvel. A nova rotina imposta pela crise sanitária suscitou mudanças no processo de trabalho e na assistência, constituindo-se desafio para o contexto municipal.

O emprego dessas tecnologias, especialmente no interior da Amazônia brasileira, requer maior atenção. Assim, as recomendações do Ministério da Saúde diante do cenário atual devem considerar as especificidades e singularidades regionais, garantindo a efetivação dos princípios do SUS. Ademais, a expansão do investimento federal em tecnologias é importante para integrar as redes de atenção básica em saúde, gerando resultados satisfatórios em relação ao "acesso, resolubilidade, integralidade e abrangência de cuidados, comodidade para as pessoas e qualificação do monitoramento de portadores de doenças crônicas já acompanhados nas unidades de saúde". ${ }^{17}$

Em relação às ações dos ACS das áreas rurais e ribeirinhas, foram desenvolvidas atividades similares aos da área urbana. Destacam-se as ações de busca ativa e organização dos moradores locais para a realização dos testes rápidos, entrega dos kits de higiene e máscaras de proteção e agendamento de consultas médicas e odontológicas, efetuadas pela equipe de Saúde da Família Fluvial. Nessas áreas, o ACS é o elo entre os moradores e o sistema de saúde, demandando atuação intensa por parte destes profissionais. Assim, a COVID-19 soma-se aos outros agravos prevalentes na população, como malária, tuberculose, violências, diarreia e outras doenças na infância. 
Embora persistam desafios relativos à atuação dos ACS no município, estes profissionais constituem-se estratégicos para o enfrentamento da pandemia e para a continuidade do cuidado. Assim, enfatiza-se a necessidade de investimentos por parte dos gestores públicos em ações de educação continuada, que tratem da temática COVID-19, visando a instrumentalização e qualificação dos ACS que atuam na linha de frente no município, especialmente aqueles pertencentes às áreas rurais.

A atual crise sanitária reforça a necessidade do fortalecimento da APS, especialmente frente às políticas de desmonte, como a nova Política Nacional de Atenção Básica $P N A B,{ }^{18,19}$ a qual reduziu quantitativamente os ACS nas equipes da ESF e integrou os Agentes de combate a endemias (ACE), fundiu competências, descaracterizou funções, redefiniu a equipe mínima, não incluindo os $A C S,{ }^{19}$ influenciado a precarização do processo de trabalho e dos atributos deste nível de atenção, principalmente em territórios vulneráveis. $^{20}$

Destaca-se a necessidade de revogação da Emenda Constitucional 95, a qual prevê o congelamento dos gastos públicos em serviços por 20 anos - incluindo os de saúde. Portanto, deve-se ampliar o financiamento da saúde durante este período de pandemia, onde existe uma alta demanda do SUS. Concomitantemente faz-se importante impedir as políticas de austeridade que têm privado a transferência de recursos para a APS. A partir de uma Atenção Primária à Saúde forte, são estruturados sistemas universais através, principalmente, de financiamento público, resultando melhorias em relação à eficiência, qualidade e equidade dos serviços prestados à comunidade. ${ }^{21,22}$

\section{Testagem em massa}

As ações de testagem em massa foram realizadas em todos os bairros do município, coordenadas pela SEMSA, conforme um cronograma previamente divulgado nos portais oficiais e em meios de comunicação local. Tais ações consistiram na busca ativa de idosos e usuários portadores de comorbidades (diabetes mellitus, hipertensão, tuberculose, doenças respiratórias, entre outras) apontadas como risco elevado para complicações em decorrência da infecção pelo novo coronavírus, incluíam procedimentos de entrevista, orientações referentes às medidas de prevenção e manejo das síndromes gripais, investigação de casos sintomáticos respiratórios/suspeitos de COVID-19, notificação e agendamento para coleta de amostras para testagem rápida domiciliar, no dia posterior a visita. Tal ação envolveu profissionais dos diversos setores do município, como saúde (ACS), desenvolvimento social e segurança pública, devidamente capacitados e equipados. 


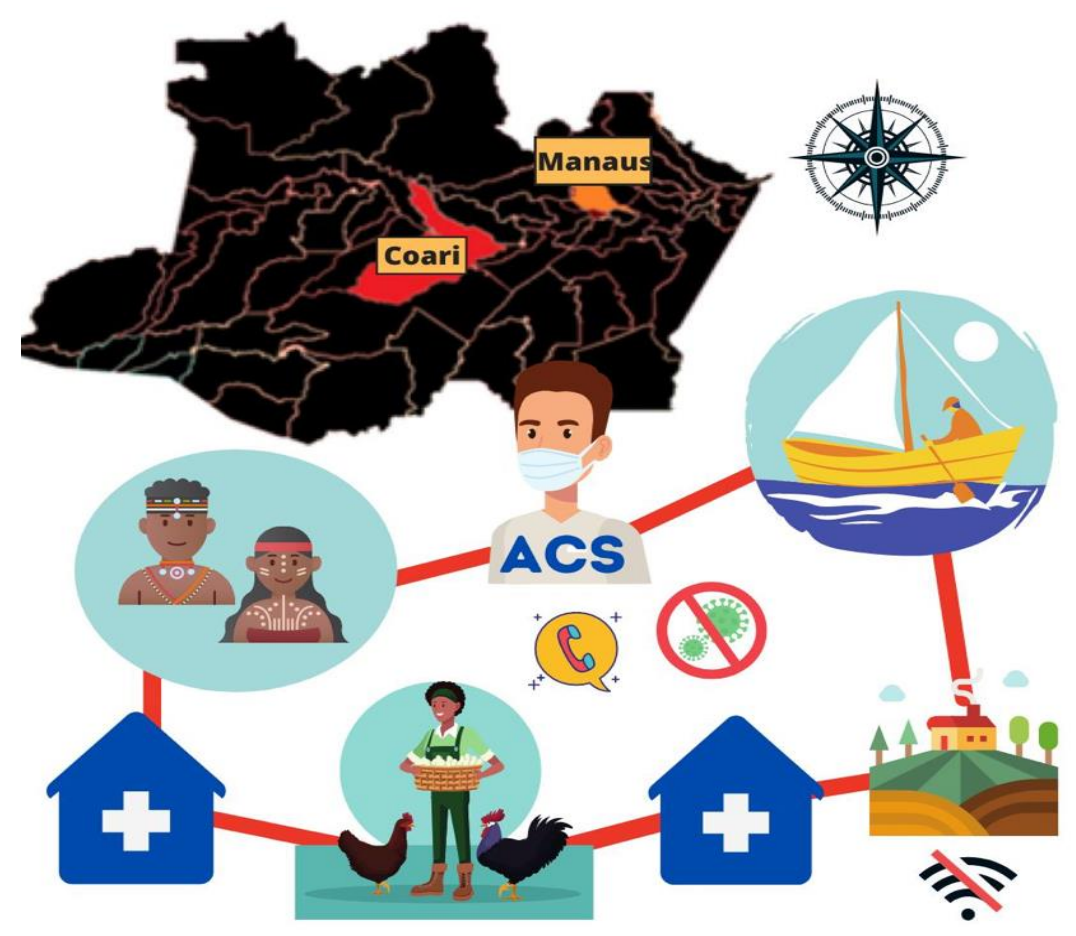

Figura 1. Representação das especificidades da Atenção Primária à Saúde no município de Coari, AM. 2020

Fonte: Elaborado pelos autores (2020).

\section{Conclusão}

Por se tratar de um município em área remota, torna-se importante destacar a relevância que a APS apresenta na assistência à população de Coari, uma vez que é este nível de atenção o responsável por maior parte dos serviços e ações de saúde. Inúmeras limitações também permearam a experiência aqui descrita, relacionando-se à disponibilidade dos serviços nos demais níveis de complexidade, recursos tecnológicos, bem como de profissionais especialistas, concentrados majoritariamente na capital. Estratégias que fortaleçam a Atenção Primária à Saúde, colocando-a no centro da agenda do governo, priorizando os recursos para seu financiamento e reconhecendo o protagonismo dos ACS para sua expansão, consolidação e qualificação, são essenciais para o sucesso do enfrentamento à COVID-19 no país, principalmente nos municípios do interior. Em Coari, os agentes comunitários de saúde (ACS) adaptaram o cotidiano de trabalho em função da pandemia, adicionando ao rol de suas atividades o monitoramento dos casos confirmados, casos suspeitos, e de seus contactantes, presencialmente ou por telefone, assumindo papel estratégico no enfrentamento da COVID-19. Por outro lado, frente às especificidades da região, com as barreiras geográficas, alta densidade domiciliar e ocorrência de doenças 
endêmicas, reforçam-se a necessidade de estratégias de instrumentalização e capacitação destes profissionais, considerando estes desafios.

\section{Referências}

1. Ministério da Saúde (BR). Painel Coronavírus. Atualizado em 14 de setembro de 2020 [citado 15 set. 2020]. Disponível em: https://covid.saude.gov.br/.

2. Floss $M$, Franco CM, Malvezzi C, Silva KV, Costa BR, Silva VXL, et al. A pandemia de COVID19 em territórios rurais e remotos: perspectiva de médicas e médicos de família e comunidade sobre a atenção primária à saúde. Cad Saude Publica. 2020;36(7):e00108920. http://dx.doi.org/10.1590/0102-311x00108920.

3. Garnelo L, Sousa ABL, Silva CO. Health regionalization in Amazonas: progress and challenges. Cienc Saude Colet. 2017;22(4):1225-34. http://dx.doi.org/ 10.1590/141381232017224.27082016.

4. Cabral ERM, Melo MC, Cesar ID, Bastos REMOTF, et al. Contribuições e desafios da Atenção Primária à Saúde frente à pandemia de COVID-19. Interamerican Journal of Medicine and Health. 2020;3:1-12.

5. Medina MG, Giovanella L, Bousquat A, Mendonça MHMD, Aquino R. Atenção primária à saúde em tempos de COVID-19: o que fazer?. Cad Saude Publica. 2020;36(8):e00149720. http://dx.doi.org/10.1590/0102-311X00149720.

6. Maciel FBM, Santos HLPC, Carneiro RAS, Souza EM, Prado NMBL, Teixeira CFS. Agente comunitário de saúde: Reflexões sobre o processo de trabalho em saúde em tempos de pandemia de Covid-19. Cien Saude Colet. 2020;25(Suppl 2):4185-95. http://dx.doi.org/10.1590/1413-812320202510.2.28102020.

7. Instituto Brasileiro de Geografia e Estatística. IBGE Cidades. Rio de Janeiro: IBGE; 2020 [citado 2 jun. 2020]. Disponível em: https://cidades.ibge.gov.br

8. Guimarães AF, Barbosa VLM, Silva MP, Portugal JKA, Reis MHS, Gama ASM. Acesso a serviços de saúde por ribeirinhos de um município no interior do estado do Amazonas, Brasil. Rev Pan Amaz Saude. 2020;11:e202000178. http://dx.doi.org/10.5123/s21766223202000178.

9. Fundação de Vigilância em Saúde do Amazonas. Boletim Epidemiológico registra 516 casos de Covid-19 neste sábado (12/09). Manaus: FVS; 2020 [citado 13 set. 2020]. Disponível em: http://www.fvs.am.gov.br/media/publicacao/12_09_20_BOLETIM_DIÁRIO_DE_CASOS_COVID-19.pdf. 
10. Ministério da Saúde (BR). Cadastro Nacional de Estabelecimentos de Saúde (CNES). Brasília: Ministério da Saúde; 2020 [citado 09 jul. 2020]. Disponível em: http://cnes.datasus.gov.br/pages/estabelecimentos/consulta.jsp.

11. Ministério da Saúde (BR). Serviço de Atenção Domiciliar. Melhor em Casa. 2020 [citado 14 set. 2020]. Disponível em: https://www.saude.gov.br/acoes-e-programas/melhor-em-casaservico-de-atencao-domiciliar/melhor-em-

casa\#: :text=0\%20Melhor\%20em\%20Casa\%20\%C3\%A9,indicada\%20para\%20o\%20seu\%2 Otratamento.

12. Daumas RP, Gulnar AS, Tasca R, Leite IC, Brasil P, Greco DB et al. O papel da atenção primária na rede de atenção à saúde no Brasil: limites e possibilidades no enfrentamento da COVID-19. Cad Saude Publica. 2020;36(6):e00104120. http://dx.doi.org/10.1590/0102$311 \times 00104120$.

13. Mendonça C, Rosset I, Gonçalves M, Molina Bastos C, Medeiros A, Dias A, et al. Resposta assistencial de um serviço docente assistencial de APS à pandemia da COVID-19. APS Rev. 2020;2(1):33-7. https://doi.org/10.14295/aps.v2i1.63.

14. World Health Organization. WHO Guideline: recommendations on digital interventions for health system strengthening. Genebra: WHO, 2020.

15. Barbosa SP, Silva AVFG. A prática da atenção primária à saúde no combate da COVID-19. APS Rev. 2020;2(1):17-9. https://doi.org/10.14295/aps.v2i1.62.

16. Ministério da Saúde (BR). Recomendações para adequação das ações dos agentes comunitários de saúde frente à atual situação epidemiológica referente ao COVID-19. Brasília, DF: Ministério da Saúde; 2020.

17. Sarti TD, Lazarini WS, Fontenelle LF, Almeida APSC. Qual o papel da atenção primária à saúde diante da pandemia provocada pela COVID-19?. Epidemiol Serv Saude. 2020;29(2):e2020166. https://doi.org/10.5123/s1679-49742020000200024.

18. Silva TL, Soares AN, Lacerda GA, Mesquita JFO, Silveira DC. Política Nacional de Atenção Básica 2017: implicações no trabalho do agente comunitário de saúde. Saude Debate. 2020;44(124):58-69. http://dx.doi.org/10.1590/0103-1104202012404.

19. Gomes CBS, Gutiérrez AC, Soranz D. Política Nacional de Atenção Básica de 2017: análise da composição das equipes e cobertura nacional da saúde da família. Cienc Saude Colet. 2020;25(4):1327-38. http://dx.doi.org/10.1590/1413-81232020254.31512019. 
20. Faria CCMV, Paiva CHA. O trabalho do agente comunitário de saúde e as diferenças sociais no território. Trab Educ Saude. 2020;18(Suppl 1):e0025183. http://dx.doi.org/10.1590/1981-7746-sol00251.

21. Starfield B, Shi L, Macinko J. Contribution of primary care to health systems and health. Milbank Q. 2005;83(3):457-502. http://dx.doi.org/10.1111/j.1468-0009.2005.00409.x.

22. Amorim DA, Mendes ÁN. Financiamento federal da atenção básica à saúde no SUS: uma revisão narrativa. J Manag Prim Health Care. 2020;12:e15.

https://doi.org/10.14295/jmphc.v12.970.

\section{Minicurrículo}

Mayline Menezes da Mata | ORCiD: 0000-0002-6142-5112

Professora do Instituto de Saúde e Biotecnologia, Universidade Federal do Amazonas, Coari, AM, Brasil.

Daiane Nascimento de Castro | ORCiD: 0000-0003-1858-9557

Professora do Instituto de Saúde e Biotecnologia, Universidade Federal do Amazonas, Coari, AM, Brasil.

Cléber Araújo Gomes | ORCiD: 0000-0002-8899-4665

Professor do Instituto de Saúde e Biotecnologia, Universidade Federal do Amazonas, Coari, AM, Brasil.

Juliberta Alves de Macêdo | ORCiD: 0000-0002-8931-7391

Professora do Instituto de Saúde e Biotecnologia, Universidade Federal do Amazonas, Coari, AM, Brasil.

Maria Helena Ribeiro De Checchi | ORCiD: 0000-0002-8431-6533

Professora do Instituto de Saúde e Biotecnologia, Universidade Federal do Amazonas, Coari, AM, Brasil.

Abel Santiago Muri Gama | ORCiD: 0000-0001-5089-6990

Professor do Instituto de Saúde e Biotecnologia, Universidade Federal do Amazonas, Coari, AM, Brasil.

Luís Paulo Souza e Souza | ORCiD: 0000-0002-9801-4157

Professor do Instituto de Saúde e Biotecnologia, Universidade Federal do Amazonas, Coari, AM, Brasil. 